Received: 2016.08 .24

Accepted: 2016.11 .02

Published: 2017.09.15

Authors' Contribution:
A Study Design
B Data Collection
C Statistical Analysis
D Data Interpretation
E Manuscript Preparation
F Literature Search
G Funds Collection

\title{
Endovascular Embolization of Renal Cell Carcinoma in a Patient with Solitary Kidney
}

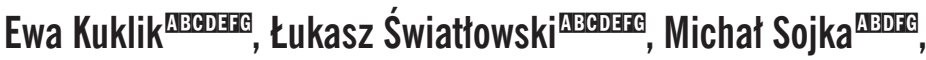 \\ Małgorzata Szczerbo-Trojanowska ${ }^{\mathrm{ABCDBFE}}$
}

Department of Radiology and Interventional Neuroradiology, Independent Public Hospital NNo. 4 (SPSK 4), Lublin, Poland

Author's address: Ewa Kuklik, Department of Radiology and Interventional Neuroradiology, Independent Public Hospital №. 4 (SPSK 4), Jaczewskieg0 8 Str., 20-001 Lublin, Poland, e-mail: ekuklik5@wp.pl

\section{Summary}

Background:

Kidney tumors account for about 3\% of tumors in adults. The primary therapy of renal cancer is the surgical removal. Traditionally, and also modern procedures are performed to remove the kidneys, especially when the tumor involves the entire kidney. In the cases of unresectable tumors embolization is used as a palliative procedure.

Case Report:

The aim of this study is to present the case of endovascular treatment of renal cell carcinoma in patient with solitary kidney. 77-years old patient had an ultrasound examination because of the pain in left lumbar region. MRI confirmed the presence of tumor size $29 \times 45 \mathrm{~mm}$ in the left kidney. The right kidney had been removed eight years earlier because of clear cell carcinoma. Histopathological diagnosis was renal clear cell carinoma. The patient did not consent to surgical treatment. Tumor embolization was proceeded as a minimally invasive procedure. Pathological tumor vessels were closed using particles filling the entire vascular tumor. Next, the blood vessels supplying the tumor were closed using a mixture of lipiodolu and glubranu. Control angiographiy of the left renal artery confirmed the effective closure of all vascular pathology. In a recent ultrasound examination which was done 15 months after surgery no evidence of vascular pathology was found.

Conclusions: Embolization of kidney cancer in particular cases may be an alternative way of treatment and give a good result in the form of stopping the growth of the tumor with simultaneous retaining the remaining parenchyma and renal function.

\author{
MeSH Keywords: $\quad$ Carcinoma, Renal Cell • Embolization, Therapeutic • Neoplastic Cells, Circulating \\ PDF file: $\quad$ http://www.polradiol.com/abstract/index/idArt/901219
}

\section{Background}

Kidney tumors account for about $3 \%$ of tumors in adults. We can distinguish several types of malignant kidney tumors of which renal cell carcinoma is the most common. It is responsible for about $75 \%$ of all malignant kidney tumors and $80 \%$ of histopathological diagnoses of renal cancer are renal clear cell carcinomas [1]. Kidney tumors are slightly more common in men than in women. The disease usually develops between 50 and 70 years of age. The primary therapy of renal cancer is surgical removal, especially when the tumor involves an entire kidney. When a kidney tumor is small, enucleation surgery is the standard treatment, which means selective excision of the tumor mass or partial nephrectomy [2].
In unresectable tumors, embolization is used as a palliative procedure. The treatment consists of catheterization of the renal artery branch that is supplying the tumor in order to achieve selective closure of the vessel. Embolization allows to reduce tumor growth, alleviate pain and inhibit possible bleeding from the tumor.

\section{Case Report}

A 77-year-old patient had an ultrasound examination because of the pain in the left lumbar region, which showed a tumor-like change in the left kidney. MRI confirmed the presence of the tumor (size $29 \times 45 \mathrm{~mm}$ ) in the lower pole of the left kidney. The right kidney had been 


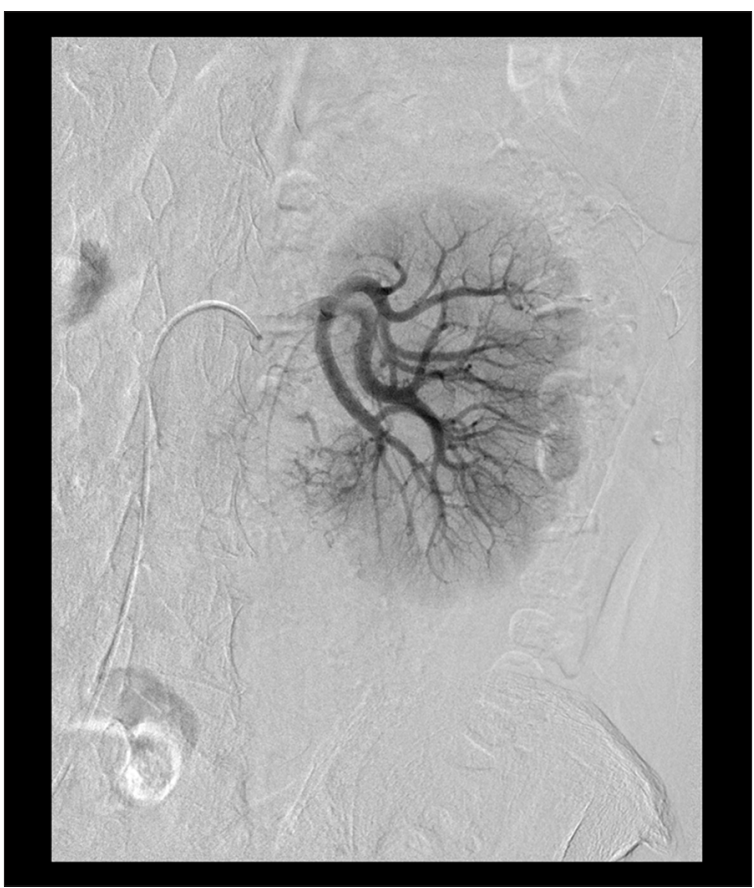

Figure 1. Angiography of the left renal artery before the procedure showing tumor mass in the lower pole of the kidney, measuring approximately $45 \times 25 \mathrm{~mm}$. Arterial phase.

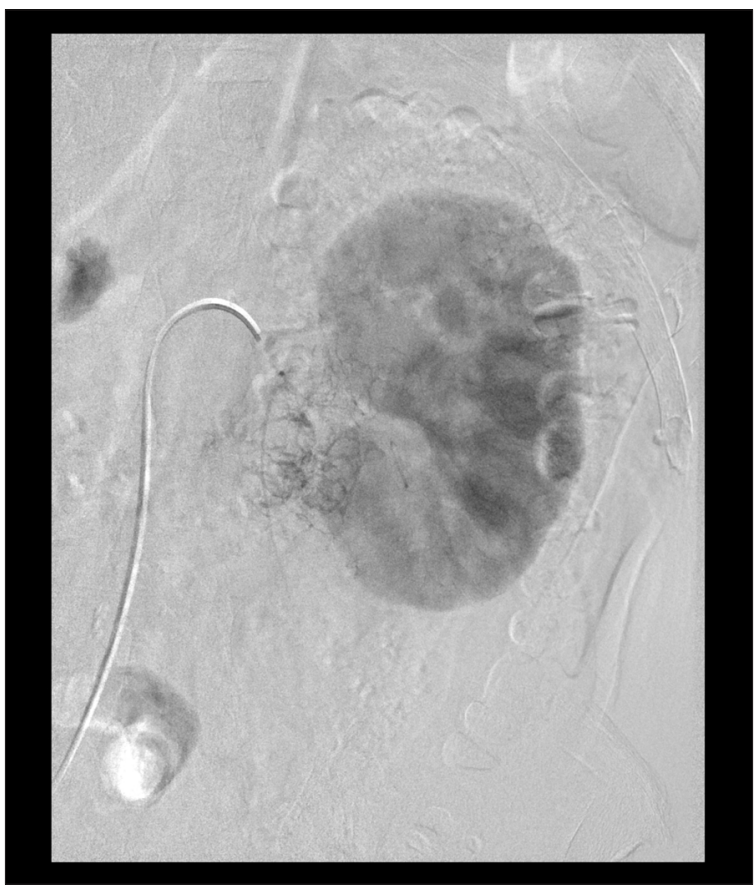

Figure 2. Angiography of the left renal artery before the procedure showing tumor mass in the lower pole of the kidney, measuring approximately $45 \times 25 \mathrm{~mm}$. Arterial phase, tissue phase.

removed eight years earlier because of clear cell carcinoma. The image of the left kidney tumor suggested a metaplastic change as an initial diagnosis. Targeted biopsy confirmed the presence of tumor that was diagnosed as renal clear cell carcinoma on a histopathological examination. The patient did not consent to surgical treatment. Tumor embolization

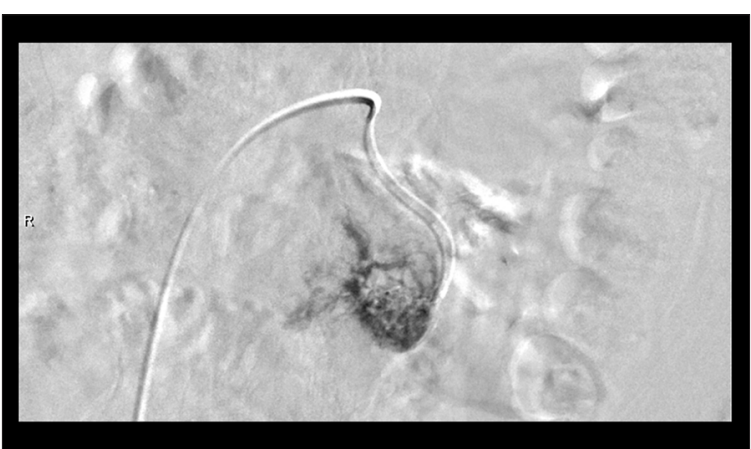

Figure 3. Selective angiography of the tumor mass.

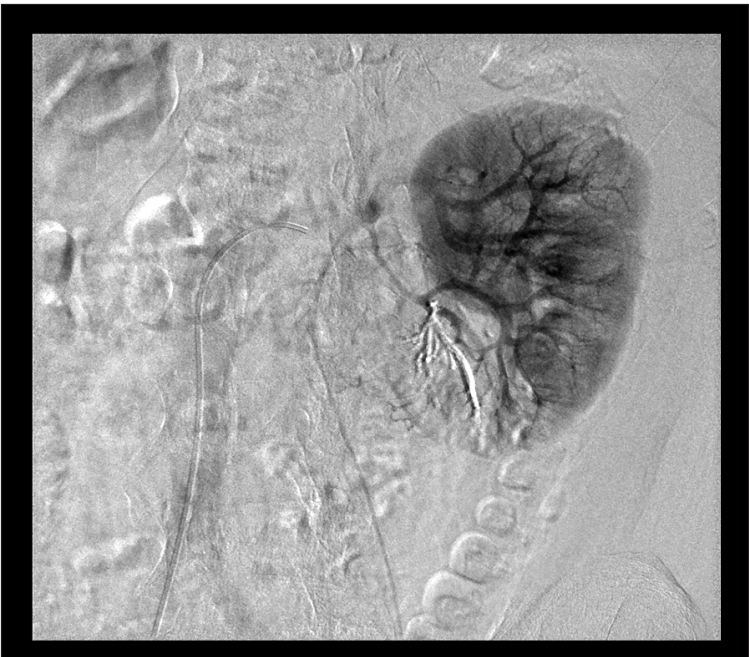

Figure 4. Control angiography of the left renal artery after the procedure showed effective closure of the vascular pathology.

was performed as a minimally invasive procedure. Firstly, a DSA examination was performed by puncturing the right femoral artery under local anesthesia using the Seldinger method. With a Levin catheter, we catheterized the left renal artery. On angiography, we visualized the tumor mass in the lower pole of the kidney, which measured approximately $45 \times 25 \mathrm{~mm}$ (Figures 1, 2). The tumor was characterized by a rich vasculature, typical for clear cell carcinoma. Then, with a type Progreate microcatheter, the small vessels supplying the tumor were selectively catheterized (Figure 3). Pathological tumor vessels were closed with Embozene 250-um particles filling the entire vascular tumor. Subsequently, the blood vessels supplying the tumor were closed using a mixture of lipiodol and glubran (in a proportion of 40-60\%). A control angiography of the left renal artery confirmed an effective closure of all vascular pathologies (Figure 4)

The remaining part of the vasculature of the kidney was normal. The course of the procedure and surgery was without complications. The patient was discharged home on the $2^{\text {nd }}$ day of hospitalization with a recommendation to undergo an ultrasound examination in a month and then every 6 months.

The ultrasound examination performed one month after the procedure showed normal renal vasculature. 
Pathological vascularization in the lower pole of left kidney was not found.

In a recent ultrasound examination, which was done 15 months after the procedure, no evidence of vascular pathology was found. The condition of the kidney did not change in comparison to the study carried out one year before (one month after embolization).

\section{Discussion}

In the early stages, renal cancer is usually asymptomatic. The typical triad of hematuria, pain and presence of a palpable tumor is seen in about $5-15 \%$ of cases. In most patients, renal cancer is discovered accidentally while performing imaging of the abdomen [3].

Renal cell carcinoma metastases spread by both the circulatory and lymphatic routes. Most metastases are seen in the lungs (50-60\%), bones (30-40\%), liver (30-40\%), lymph nodes $(36 \%)$ and brain (5\%) [4]. Bilateral kidney tumors are extremely rare and are represent in only about $1-3 \%$ of cases [5].

Almgard was the first to popularize embolization of renal tumors in the 1970s. Indications for renal artery embolization for the treatment of renal tumors include supporting treatment before nephrectomy, palliative therapy and treatment of angiomyolipomas [2].

Nurmi et al. described a group of 25 patients with renal cancer who were treated with embolization of the renal artery. The aim of the procedure was to stop bleeding from the urinary tract and to alleviate pain. An effective inhibition of bleeding was achieved in 11 of the 14 patients with bleeding, and pain disappeared in $50 \%$ of cases. There was no prolongation of survival. All patients who underwent follow-up died of renal cancer within 38 months. In one patient, ethanol embolization was complicated by necrosis of the colon [6].

In the scientific literature, there are reports on the immunomodulatory effects of radioembolization (RAE). Zielinski et al. describe the use of this procedure in 234 patients with kidney cancer. One-hundred-eighteen subjects underwent preoperative embolization and then nephrectomy, and the remaining 116 subjects underwent only nephrectomy. The overall 5-year and 10-year survival rates were as follows: $62 \%$ and $47 \%$ in the group treated with preoperative embolization, and only $35 \%$ and $23 \%$ in the group treated surgically, respectively [7].

In recent years, there have been more and more reports on the treatment of renal tumors by radiofrequency ablation (RFA). It results in the destruction of the tumor with radiofrequency waves under the guidance of MRI, CT and

\section{References:}

1. Borówka A, Szcześniak C: Zalecenia postępowania diagnostycznego i terapeutycznego w raku nerki. Współczesna Onkologia, 2004; 8: 181-88 [in Polish]

2. http://onkologia.org.pl [in Polish] ultrasound. Initially, radiofrequency ablation was used in the treatment of primary and metastatic tumors of the liver. The first use of radiofrequency ablation for the treatment of renal tumors was described in 1997 [8].

Since then, many studies have been published that have demonstrated the efficacy of ablation for the treatment of kidney tumors with a simultaneous maintenance of kidney function. Best et al. reported of the use of radiofrequency ablation as a primary treatment for renal tumors. The treatment was performed in 159 patients with renal tumors, including 138 patients with a histopathological diagnosis of clear cell carcinoma. The results showed a longer survival time in patients with tumors that had a diameter smaller than $3 \mathrm{~cm}$, and 3-year and 5-year survival times were $96 \%$ and $95 \%$, respectively. In tumors with a diameter larger than $3 \mathrm{~cm}, 3$-year and 5-year average survival times were $79 \%$ and $79 \%$, respectively [9]. Soo Dong Kim et al. described percutaneous radiofrequency ablation for the treatment of renal tumors in 36 patients. During 49 months of follow-up, local recurrence occurred in 3 patients, which was most likely due to technical imperfections of the intervention with incomplete ablation of the tumor. Patients with a known cancer recurrence underwent re-ablation of the tumor with satisfactory results [10]. The efficacy of radiofrequency ablation in the long-term observation was also presented. The results of the publication indicate that the procedure is effective, also in the long-term observation. Good treatment outcomes depend on appropriate qualification of patients, and the main indicator of the effectiveness of treatment is tumor size. The best results are obtained with small tumors, up to $3 \mathrm{~cm}$ in diameter [9]. In our case, the clear cell tumor of the left kidney could be treated as a metastatic change or a new primary lesion. Eight years before, when the right kidney was removed because of cancer, the image of the left kidney was normal. Because of lack of patient consent for surgery and the inability to perform thermoablation, we decided to treat the kidney tumor by superselective embolization of the pathological vessels of the tumor. So far, we have not found any cases of embolization of solitary kidney tumors as the initial and only treatment. This procedure is not a standard treatment. However, observation of this patient indicates that the embolization of kidney cancer in certain cases may be an alternative way of treatment and could give good results by inhibiting tumor growth with a simultaneous maintenance of function of the remaining renal parenchyma.

\section{Conclusions}

Embolization of kidney cancer in certain cases may be an alternative way of treatment and could give good results by inhibiting tumor growth with a simultaneous maintenance of function of the remaining renal parenchyma.

3. Li D, Pua BB, MD, Madoff DC: Role of embolization in the treatment of renal masses. Semin Intervent Radiol, 2014; 31(1): 70-81

4. Thyavihally Y, Mahantshetty U, Chamarajanagar R et al:

Management of renal cell carcinoma with solitary metastasis. World J Surg Oncol, 2005; 3: 48 
5. Motzer R, Bander N, Nanus D: Renal-cell carcinoma. N Eng J Med, 1996; 12: 865-75

6. Nurmi M, Satokari K, Puntala P: Renal artery embolization in the palliative treatment of renal adenocarcinoma. Scand J Urol Nephrol, 1987; 21(2): 93-96

7. Zielinski H, Syrylo T, Szmigielski S: Renal Artery embolization in treatment of renal cancer with emphasis on response of immune system. In: Renal tumor. Chen J. (ed.), InTech, 2013; Chapter 6
8. Zlotta AR, Wildschutz T, Raviv G et al: Radiofrequency interstitial tumor ablation (RITA) is a possible new modality for treatment of renal cancer: Ex vivo and in vivo experience. J Endourol, 1997; 11: 251-58

9. Best SL, Park SK, Youssef RF, Olweny EO: Long-term outcomes of renal tumor radio frequency ablation stratified by tumor diameter: Size matters. J Urol, 2012; 187(4): 1183-89

10. Kim SD, Yoon SG, Sung GT: Radiofrequency ablation of renal tumors: Four-year follow-up results in 47 patients. Korean J Radiol, 2012; 13(5): $625-33$ 